\title{
Beam dynamics of the Neutralized Drift Compression Experiment-II (NDCX-II), a novel pulse-compressing ion accelerator
}

\author{
A. Friedman, ${ }^{*}$ J. J. Barnard, R. H. Cohen, D. P. Grote, S. M. Lund, and W. M. Sharp \\ Lawrence Livermore National Laboratory, Livermore, CA 94550, USA \\ A. Faltens, E. Henestroza, J.-Y. Jung, J. W. Kwan, E. P. Lee, \\ M. A. Leitner, B. G. Logan, J.-L. Vay, and W. L. Waldron \\ Lawrence Berkeley National Laboratory, Berkeley, CA 94720, USA \\ R. C. Davidson, M. Dorf, E. P. Gilson, and I. D. Kaganovich \\ Princeton Plasma Physics Laboratory, Princeton, NJ 08543, USA
}

(Heavy Ion Fusion Science Virtual National Laboratory collaboration)

(Dated: December 19, 2009)

\begin{abstract}
Intense beams of heavy ions are well suited for heating matter to regimes of emerging interest. A new facility, NDCX-II, will enable studies of warm dense matter at $\sim 1 \mathrm{eV}$ and near-solid density, and of heavy-ion inertial fusion target physics relevant to electric power production. For these applications the beam must deposit its energy rapidly, before the target can expand significantly. To form such pulses, ion beams are temporally compressed in neutralizing plasma; current amplification factors of 50-100 are routinely obtained on the Neutralized Drift Compression Experiment (NDCX) at LBNL. In the NDCX-II physics design, an initial non-neutralized compression renders the pulse short enough that existing high-voltage pulsed power can be employed. This compression is first halted and then reversed by the beam's longitudinal space-charge field. Downstream induction cells provide acceleration and impose the head-to-tail velocity gradient that leads to the final neutralized compression onto the target. This paper describes the discrete-particle simulation models (1-D, 2-D, and 3-D) employed and the space-charge-dominated beam dynamics being realized.
\end{abstract}

\section{INTRODUCTION}

Intense beams of heavy ions are well suited for heating matter to regimes of emerging interest. A new facility, the second Neutralized Drift Compression Experiment (NDCX-II), will enable studies of heavy-ion beam-heated matter in the poorly-understood "warm dense matter" (WDM) regime at $\sim 1 \mathrm{eV}$ and near-solid density. In addition, NDCX-II will enable studies of ion energy deposition into an ablating plasma, physics that is relevant to inertial fusion directly driven by ion beams. The project is being carried out by the Heavy Ion Fusion Science Virtual National Laboratory (HIFS-VNL), a collaboration of Lawrence Berkeley National Laboratory, Lawrence Livermore National Laboratory, and the Princeton Plasma Physics Laboratory. For background information on this program, see Ref. 1.

The above-mentioned studies require that the beam deposit its energy rapidly, before the target can expand significantly. This need for rapid target heating motivated the development of ion beam "drift compression" in a neutralizing plasma. In this process, a head-to-tail velocity gradient ("tilt") is imparted to the beam, causing it to shorten as it travels ("drifts"). So long as the plasma electron density exceeds the ion beam density, the plasma electrons can respond so as to reduce very effectively the space-charge forces that otherwise would limit the compression. Current amplification factors of $\sim 50-100$ are now routinely generated, using $\mathrm{K}^{+}$ions at
300-400 keV, on the Neutralized Drift Compression Experiment (NDCX) at LBNL. ${ }^{2}$

The construction of an improved research platform, NDCX-II, is underway at LBNL. It will be the first ion induction accelerator designed to heat targets using short pulses. NDCX-II will use refurbished and modified induction cells, Blumlein voltage sources, and transmission lines from the decommissioned Advanced Test Accelerator (ATA) electron induction linac at LLNL. An initial 15-cell configuration is due to be completed in 2012; assuming excellent final neutralization, it is projected to deliver at the target plane a sub-ns 10 Ampere pulse of 1.5-2 $\mathrm{MeV} \mathrm{Li}^{+}$ions focused to a sub-mm spot. NDCX-II is designed to be extensible and reconfigurable, and we anticipate exploiting those attributes by adding cells to achieve higher energies and a variety of pulse shapes.

The Bragg peak of the energy deposition rate for Lithium ions on an Aluminum foil target falls at $\sim 1.9 \mathrm{MeV}$. An attractive operating point for a number of WDM studies would employ ions entering the target with a mean kinetic energy of $2.8 \mathrm{MeV}$, heating it uniformly while slowing through the Bragg peak and depositing most of its energy. The studies presented here primarily concern a 22 cell configuration, in which a $\sim 500 \mathrm{~ns}$ pulse of $\mathrm{Li}^{+}$ions is compressed to $\sim 1 \mathrm{~ns}$ as it is accelerated it to $\sim 3.5 \mathrm{MeV}$ over $\sim 10 \mathrm{~m}$. This compression is accomplished in two stages: the accelerator itself, which shortens the pulse from $\sim 1 \mathrm{~m}$ to $\sim 0.2 \mathrm{~m}, 20 \mathrm{~ns}(\sim 5 \mathrm{x}$ increased speed and $\sim 5 \mathrm{x}$ decreased length); and a down- 


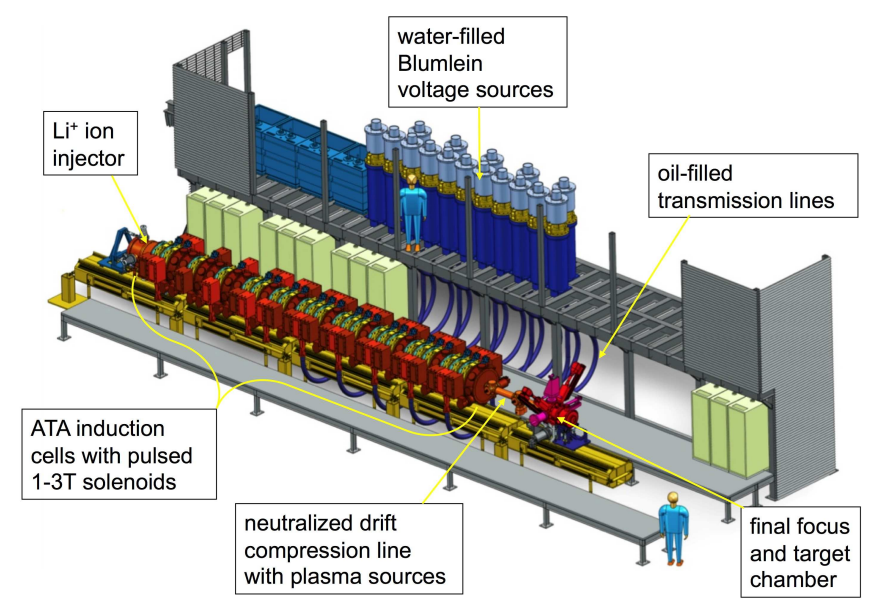

FIG. 1: (color online) NDCX-II layout for 23 induction cells.

stream neutralized drift compression line, which enables the final compression (spatial and temporal) through a factor of 20 or more. A similar 23-cell layout is shown in Fig. 1. We have also examined systems with more cells and thus greater final kinetic energies; these can be realized via straightforward extension of the machine.

The ferrimagnetic cores in each ATA cell each offer $0.017 \mathrm{~V}$-s of flux swing; the Blumleins can source as much as $250 \mathrm{kV}$ with a full-width at half-maximum of 70 ns. Passive pulse-shaping elements can be inserted into the "compensation boxes" attached to each cell, offering some flexibility in the acceleration waveforms. Custom pulsers are needed to generate longer pulses at the front end of the machine, and cost considerations motivate limiting the voltage of these to $100 \mathrm{kV}$. The $0.28-\mathrm{m}$ NDCX-II lattice period is unchanged from that of the ATA; the acceleration gaps (across which the driving electric field appears) are $2.8 \mathrm{~cm}$ long. Because ions do not rapidly reach high speeds, transverse confinement of the beam against space charge forces requires that the 0.3-T DC solenoids from ATA, which were adequate for electrons, be replaced by pulsed solenoids with fields of order 2-3 T.

For three reasons it is necessary to rebuild parts of the ATA cells. First, a major reduction of the Volt-seconds available for acceleration results when the return flux of a pulsed solenoid passes through a core and saturates it at least locally. To prevent such reduction, we use a solenoid of smaller radius than the original and insert a copper layer outward of it, in order to channel the flux so that it returns through the $\sim 5 \mathrm{~cm}$ space between the solenoid and the copper. Second, an acceleration gap must be "on" while any of the beam (excepting its tenuous ends) overlaps the fringe electric field, which we consider in the ASP code (described below) to extend halfway to the next gap; the transit time for a single particle across this field can be comparable to the time for the beam to pass a fixed axial position. To shorten this fringe, it is again necessary to reduce the pipe radius (at least at the acceleration gaps). To these ends, the original $6.72 \mathrm{~cm}$ ATA beam-pipe bore is reduced to $3.97 \mathrm{~cm}$ throughout the machine. Finally, the end plates of the ATA cells will be made thinner near the axis, to reduce the field penetration time and the energy wasted by eddy currents.

The NDCX-II beam is space-charge-dominated both longitudinally and transversely, and the physics design exploits longitudinal space-charge forces in a fundamental way. An initially imposed (non-neutralized) compression renders the pulse short enough $(\lesssim 70 \mathrm{~ns})$ for efficient use of the downstream induction cells. This initial compression is first halted and then reversed by the space charge field. The downstream Blumlein-powered cells impose most of the acceleration as well as the head-to-tail velocity gradient that enables the final neutralized drift compression onto the target.

We are using analysis, an interactive 1-D PIC code (ASP) with optimizing capabilities and a centroid-offset model, as well as both axisymmetric and 3-D Warp ${ }^{3}$ simulations to develop the NDCX-II accelerator. This paper describes the models that were developed for these studies, and the physics design that has resulted from their application; see also Refs. 4-7. Section II describes the ASP code, which serves as our principal tool for design synthesis. Section III describes the longitudinal beam dynamics of NDCX-II. Section IV describes axisymmetric Warp simulation studies used to develop the transverse beam confinement design, and 3-D studies of misalignments and the mitigation of their effects carried out using both ASP and Warp. Final comments are offered in Section V.

\section{ONE-DIMENSIONAL ASP CODE MODEL}

The ASP code ("Acceleration Schedule Program") was developed to facilitate the synthesis and optimization of NDCX-II acceleration schedules. The code uses wellestablished particle-in-cell methods to follow the beam's evolving $\left(z, v_{z}\right)$ phase space, where $z$ is the longitudinal coordinate and $v_{z}$ the corresponding velocity, but it solves for the space-charge field with a multiscale 1-D Poisson formulation, ${ }^{8}$ as follows.

We begin with the long-wavelength limit of the longitudinal space-charge field. The Poisson equation $\nabla^{2} \phi(r, z)=-\rho(r, z) / \epsilon_{0}$ relates the electrostatic potential $\phi$ to the beam charge density $\rho$, and in cylindrical coordinates its terms satisfy $\left|\partial^{2} \phi / \partial z^{2}\right| \ll|(1 / r) \partial / \partial r(r \partial \phi / \partial r)|$. Thus, at any value of $z,(1 / r) \partial / \partial r(r \partial \phi / \partial r) \approx-\rho(r) / \epsilon_{0}$; integrating, $\partial \phi / \partial r=-\lambda_{\text {enc }}(r) / 2 \pi \epsilon_{0} r$, where $\lambda_{\text {enc }}(r)$ is the beam charge per unit length enclosed within radius $r$. For simplicity, assume $\rho(r)=\rho_{0}$ for $r<r_{b}$ and $\rho(r)=0$ for $r_{b} \leq r<r_{w}$, where $r_{b}$ and $r_{w}$ are respectively the beam and wall radii. Then we have $\lambda_{\text {enc }}(r)=\lambda \min \left\{1,\left(r / r_{b}\right)^{2}\right\}$, where $\lambda=\lambda(z)$ is the to- 
tal line-charge density, and integration yields

$$
\phi=\frac{\lambda}{2 \pi \epsilon_{0}} \begin{cases}{\left[\frac{1}{2}\left(1-\frac{r^{2}}{r_{b}^{2}}\right)+\ln \frac{r_{w}}{r_{b}}\right],} & r<r_{b} \\ \ln \left(\frac{r_{w}}{r}\right), & r_{b} \leq r<r_{w}\end{cases}
$$

and the electric field $E_{z}=-\partial \phi / \partial z$ within the beam is

$$
\begin{aligned}
E_{z}(r, z)=-\frac{1}{2 \pi \epsilon_{0}}\{ & {\left[\frac{1}{2}\left(1-\frac{r^{2}}{r_{b}^{2}}\right)+\ln \frac{r_{w}}{r_{b}}\right] \frac{\partial \lambda(z)}{\partial z} } \\
& \left.-\left[1-\frac{r^{2}}{r_{b}^{2}}\right] \frac{\lambda(z)}{r_{b}} \frac{\partial r_{b}}{\partial z}\right\} .
\end{aligned}
$$

For a space-charge-dominated beam (denoted by the subscript "scd" in the following) in a transport line of uniform transverse focusing strength, $\rho$ remains constant while $r_{b}$ grows as the pulse is compressed: $\lambda / r_{b}^{2}=$ constant, $\partial \lambda / \partial z=\left(2 \lambda / r_{b}\right) \partial r_{b} / \partial z$, and

$$
E_{z}(r, z)=-\frac{g_{\mathrm{scd}}}{4 \pi \epsilon_{0}} \frac{\partial \lambda(z)}{\partial z} ; \quad g_{\mathrm{scd}}=2 \ln \frac{r_{w}}{r_{b}}
$$

where $g_{\text {scd }}$ is the geometrical or "g"-factor for this case.

For an emittance-dominated (subscript "ed") beam in a uniform transport line, $r_{b}$ does not change as the pulse is compressed. Using Eq. (2) and averaging over the beam cross-section, noting $\left\langle r^{2} / r_{b}^{2}\right\rangle=1 / 2$,

$$
\left\langle E_{z}\right\rangle(z)=-\frac{g_{\mathrm{ed}}}{4 \pi \epsilon_{0}} \frac{\partial \lambda(z)}{\partial z} ; \quad g_{\mathrm{ed}}=2 \ln \frac{r_{w}}{r_{b}}+\frac{1}{2} .
$$

In ASP we solve, in finite difference form, the crosssection-averaged field equations

$$
\frac{\partial^{2} \phi(z)}{\partial z^{2}}-k_{\perp}^{2} \phi(z)=-\frac{\lambda(z)}{\epsilon_{0} \pi r_{b}^{2}} ; \quad E_{z}(z)=-\frac{\partial \phi(z)}{\partial z}
$$

where the transverse inverse scale length $k_{\perp}$ is given by

$$
k_{\perp}^{2}=4 /\left(g_{0} r_{b}^{2}\right) .
$$

For short-wavelength longitudinal variations the $k_{\perp}^{2}$ term in Eq. (5) is relatively small, while for long wavelength variations $E_{z}$ approaches the correct limit for a g-factor of $g_{0}$. NDCX-II will not use a uniform transport line; the solenoid strengths will be set individually to establish a more nearly constant beam radius, and the ASP user specifies a corresponding nominal beam radius $r_{b}$. Thus, $g_{0}$ falls between $g_{\text {scd }}$ and $g_{\text {ed }}$, and is in practice tuned to obtain agreement with $(r, z)$ Warp calculations.

ASP represents the accelerating-gap fields $E_{z, i}(z, t)$ due to each time-varying gap voltage $V_{i}(t)$ via the "Lee" model ${ }^{9}$ we use the single-term approximation (Eq. A7 of the reference) because the gap is narrow.

The general "type" of each accelerating waveform (including ideal ramps or flat-tops, circuit-models of various kinds, and self-adjusting waveforms that level the beam energy or counteract beam end expansion) is set by the user. The code automatically adjusts each waveform using estimated times of beam entry into and exit from the gap's fringe field, constrained by user-specified limits to peak voltage and Volt-seconds.

In addition to the longitudinal coordinates $\left(z_{k}, v_{z k}\right)$ of each simulation particle (beam slice) $k$, ASP can also track the transverse coordinates $\left(x_{k}, y_{k}, v_{x k}, v_{y k}\right)$ of its centroid. In a perfectly aligned system, these all would remain zero. However, misalignments drive the beam off-axis. Furthermore, because of the head-to-tail energy variation along the beam, once a displacement occurs the individual beam slices gyrate in the solenoid fields at their own particular rates. For solenoids of uniform polarity, the result is a "corkscrew" distortion. ${ }^{10}$ Fortunately, methods for minimizing its amplitude have been developed for electron linacs, ${ }^{11}$ and our studies (described below) show that they remain effective for NDCX-II despite its large energy variation.

ASP was designed to be interactive; it is written in the Python scripting language, with a few computationallyintensive routines in Fortran. A few hundred simulation particles are used, and a run without optimization takes less than a minute on a single processor. Optimizations of two kinds are routinely carried out: variations of the applied voltage waveforms $V_{i}(t)$ to establish the acceleration schedule, and tuning of the "steering" dipole magnet strengths to minimize the beam's corkscrew and off-axis shift. On a $2.2 \mathrm{GHz}$ AMD processor, each "steering" run takes between two and three hours.

In order to derive initial conditions for the ASP beam, we begin by carrying out a series of axisymmetric $(r, z)$ Warp simulations of the source diode and injector, iterating until we arrive at an attractive design. We then record the beam's parameters as it passes through a plane upstream of the first gap, and import that data into ASP. We run ASP iteratively, to develop an optimized combination of accelerating waveforms and cell placements such as is described in Section III. This configuration is then transferred to Warp, and a series of $(r, z)$ runs carried out to arrive at a set of field strengths for the solenoids in the accelerator that yield a transversely "well-matched" beam, and at an optimized final-focus configuration wherein the focal plane coincides with the plane of shortest pulse duration. At this point, we can study three-dimensional effects. We import the solenoid strengths into ASP, and use that code's centroid model to study misalignment effects and beam steering techniques; we also run Warp in 3-D mode to assess the effects of misalignments on the focal intensity. Fully integrated Warp simulations incorporating beam sensing and steering (including noise and errors) are yet to be done.

\section{LONGITUDINAL DYNAMICS OF NDCX-II}

NDCX-II requires a compact and inexpensive design that makes good use of the ATA hardware. To that end we have identified two principles which serve as guides to development of an effective acceleration schedule. The first of these is to "shorten the beam first." We compress 


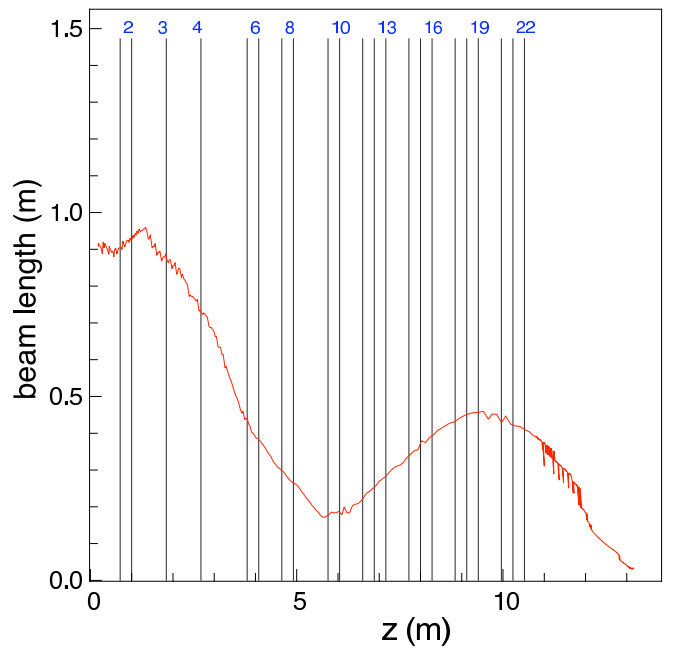

FIG. 2: Pulse length vs. $z$. Vertical lines denote acceleration gaps, some of which are numbered.

it longitudinally before the main acceleration via a process of (non-neutralized) drift compression. We use the initial cells to "equalize" the beam energy (that is, make the mean kinetic energy more nearly independent of $z$ ), impose a velocity tilt, and confine the beam; space is left for drift compression. The goal is to achieve a sub-70 ns beam transit time through the acceleration gaps (including their fringe fields) using as few cells as possible, so that we can maximize the number of Blumlein-driven $200-250 \mathrm{kV}, 70 \mathrm{~ns}$ pulses. It is necessary to compress the beam carefully so as to minimize the effects of nonuniform space charge. Thus we seek to achieve (ideally) a large linear velocity tilt with $v_{z}(z) \propto z$ and a smooth density profile. We obtain the desired waveforms using a least-squares optimization that penalizes both nonlinearity and nonuniformity.

The second design principle is to "let the beam bounce." Rapid inward motion in the beam frame is required to reduce the pulse duration below $70 \mathrm{~ns}$. Space charge ultimately inhibits this compression. At that point, the beam is shorter than the fringe field length, and it is not practical to sustain such a compressed bunch. The beam "bounces" and starts to lengthen. Nonetheless, the duration can remain below $70 \mathrm{~ns}$ if the beam is accelerated rapidly enough with flat-top pulses. Ramped voltages in the final gaps impose the velocity tilt for neutralized drift compression onto the target.

Figures 2 and 3 show the evolution of the beam length and duration. The first eight cells are driven by new pulsers and the rest by ATA Blumlein sources. Note in Fig. 3 how the time for the entire beam to cross a gap with its fringe field is rapidly brought below $70 \mathrm{~ns}$ and thereafter remains below that cutoff.

Most of the required waveforms (shown in Fig. 4) are simple enough to be formed with passive circuits in the "compensation boxes" attached to the ATA cells. While ASP can solve simplified circuit equations for a variety of pulse shapes, laboratory tests have demonstrated a

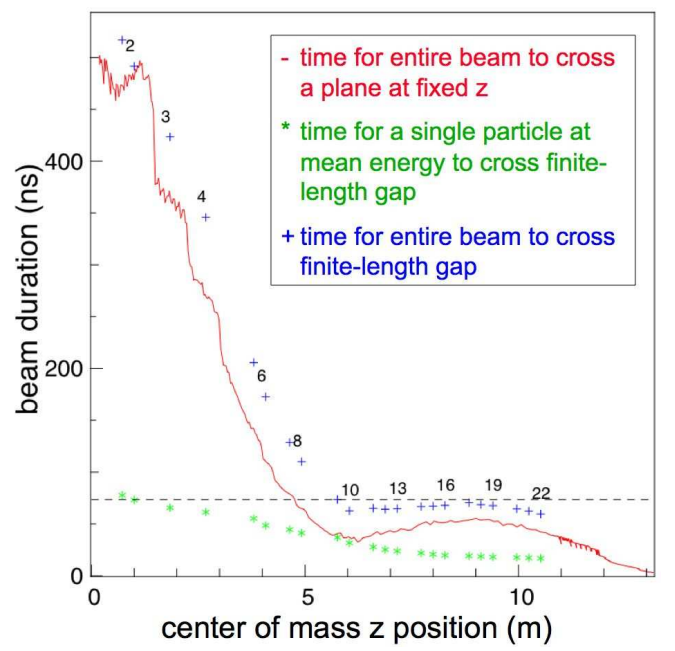

FIG. 3: (color online) Pulse duration vs. $z$.

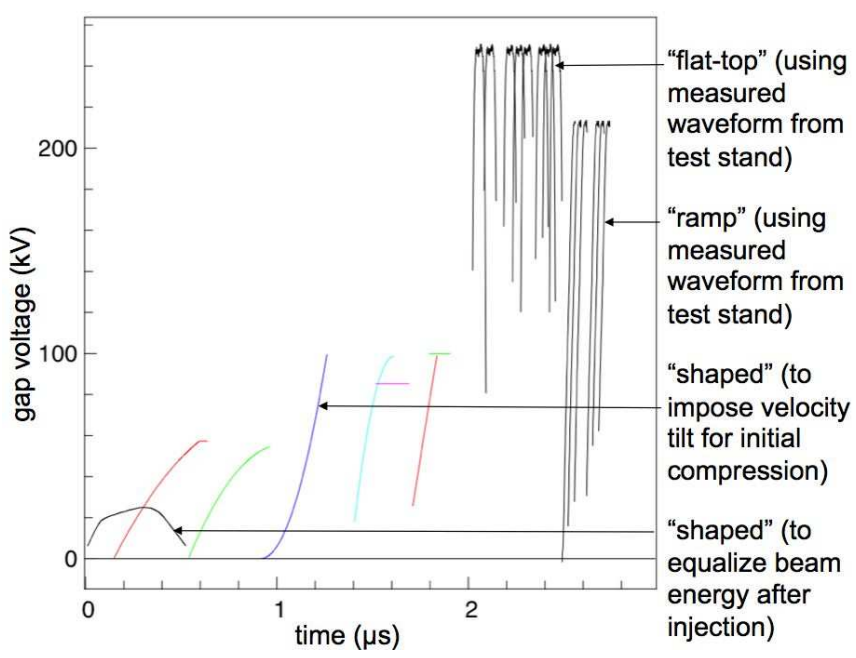

FIG. 4: (color online) Waveforms for acceleration from ASP.

high-quality $\sim 60 \mathrm{~ns}$ ramp, and most of our simulations now use that experimental data as well as the measured unmodified $250-\mathrm{kV}$ flat-top waveforms.

Figure 5 shows the evolution of the phase space and current. The final panel shows the beam when its centroid is at the plane of best longitudinal focus. This plane is estimated by an RMS measure ${ }^{12}$ and refined by searching for that plane through which the most current flows in a 1-ns window. In ASP, the beam is assumed perfectly neutralized after it exits the accelerator (the space-charge field is ramped to zero over a user-specified length).

\section{MULTIDIMENSIONAL STUDIES}

As mentioned earlier, we are carrying out both $(r, z)$ and full 3-D simulations of NDCX-II using the Warp code. These multidimensional studies are essential in view of the need to accelerate and control as large a total charge as feasible. For focusing elements (here, solenoids) of given strength and bore, and for a beam of 

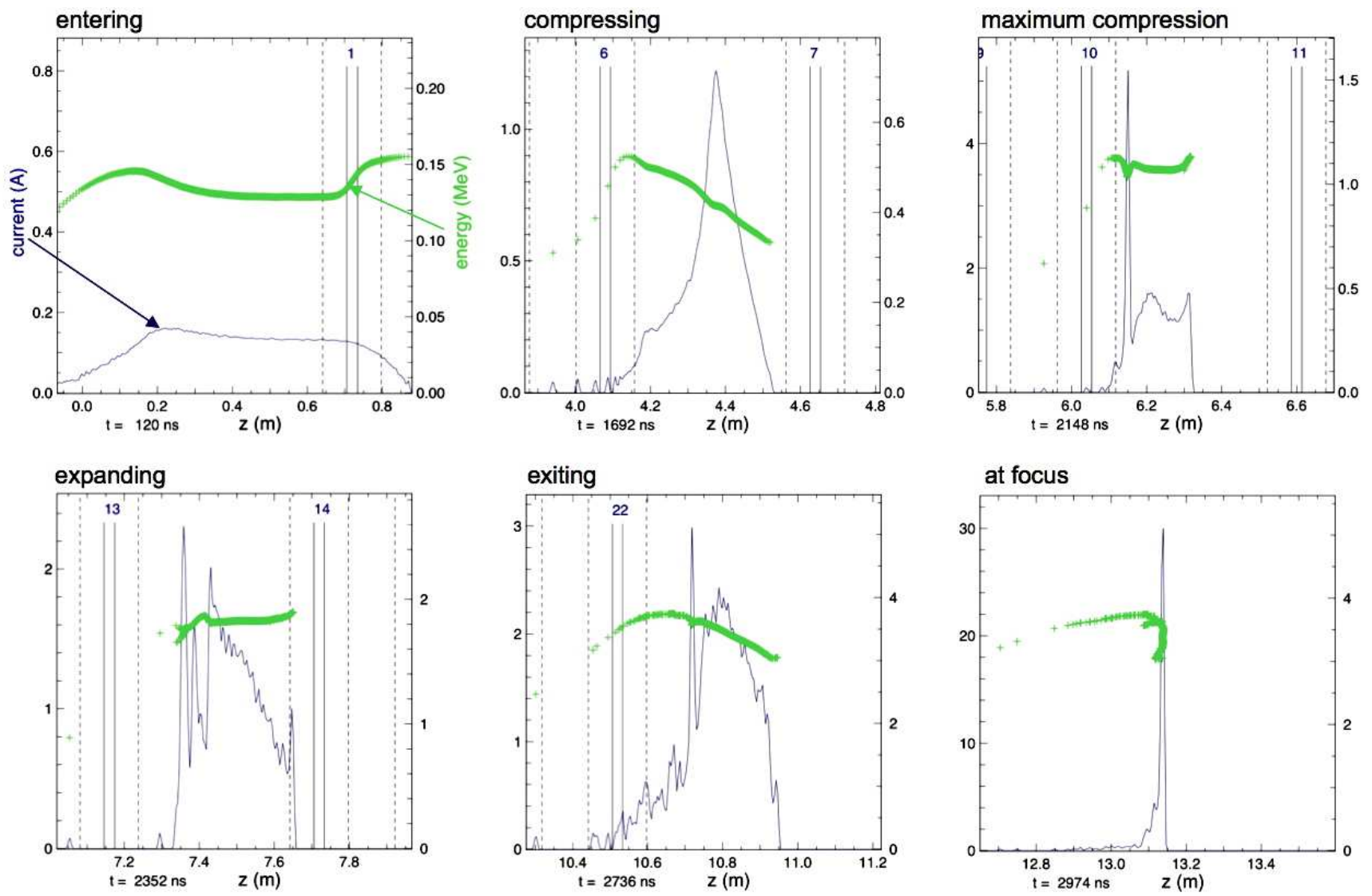

FIG. 5: (color online) Snapshots from an ASP simulation, showing evolution of kinetic energy and current profiles.

given composition and kinetic energy, the need for transverse confinement limits the transportable line charge density. Solenoids were chosen as the confinement system for NDCX-II, since they, in contrast with quadrupoles, can handle the large line-charge density at the "bounce." As is evident above, the ability to confine a beam longitudinally via shaped voltage pulses is not unlimited. Furthermore, practical injectors impose limitations on the beam current, and practical beam lines have imperfections of various kinds that can lead to dilution of the phase-space density (emittance growth). Finally, focusing onto a target is itself constrained by aberrations and practical limitations. Thus, detailed multidimensional simulations are essential to the design process.

In these studies, we use Warp's electrostatic models; this choice is justified because the ion velocities are low and because we are simulating an almost entirely onecomponent system. The effects of stray "electron clouds" are expected to be minimal because the applied acceleration fields are far stronger than the beam fields and readily sweep electrons upstream and out of the beam. For these design studies, we do not model the neutralization process, and instead merely assume ideal neutralization in the drift-compression line and beyond. Extensive studies of the neutralization process and of beam dynamics in the presence of plasma have been reported elsewhere. ${ }^{13-17}$
Initially, both steady-flow and time-dependent $(r, z)$ models are used to design the injector (Fig. 6). For scoping, we position one refinement patch at the emitter; to obtain converged voltage waveforms we will use full multi-level Adaptive Mesh Refinement. ${ }^{18}$ Our injector simulations have assumed emission densities ranging from 1 to $3 \mathrm{~mA} / \mathrm{cm}^{2}\left(2 \mathrm{~mA} / \mathrm{cm}^{2}\right.$ in the case presented here); this range has been measured on our test stand. A shortcoming of our modeling is that the Warp runs assume space-charge-limited flow from the emitting surface. However, the experimental data indicate that the emission of $\mathrm{Li}^{+}$ions does not follow space-charge-limited scaling for current densities above $0.2-1 \mathrm{~mA} / \mathrm{cm}^{2}$, depending upon the source temperature. ${ }^{19}$ We are developing a computational model that matches the measured data, and will adjust the injector design appropriately.

These simulations include detailed acceleration gap and solenoid field models, and solve for the beam selffield in a conducting pipe. The gap fields have a twodimensional variation (the fringe field has reduced axial extent at larger radius); we obtain these fields by scaling from a separate, preliminary calculation in which we solve Laplace's equation for an effective electrostatic potential, with boundary values on the pipe wall at each side of the gap that differ by a unit applied voltage. The line integral of the physical electric field around a closed path that includes the machine's axis and its outer wall is 

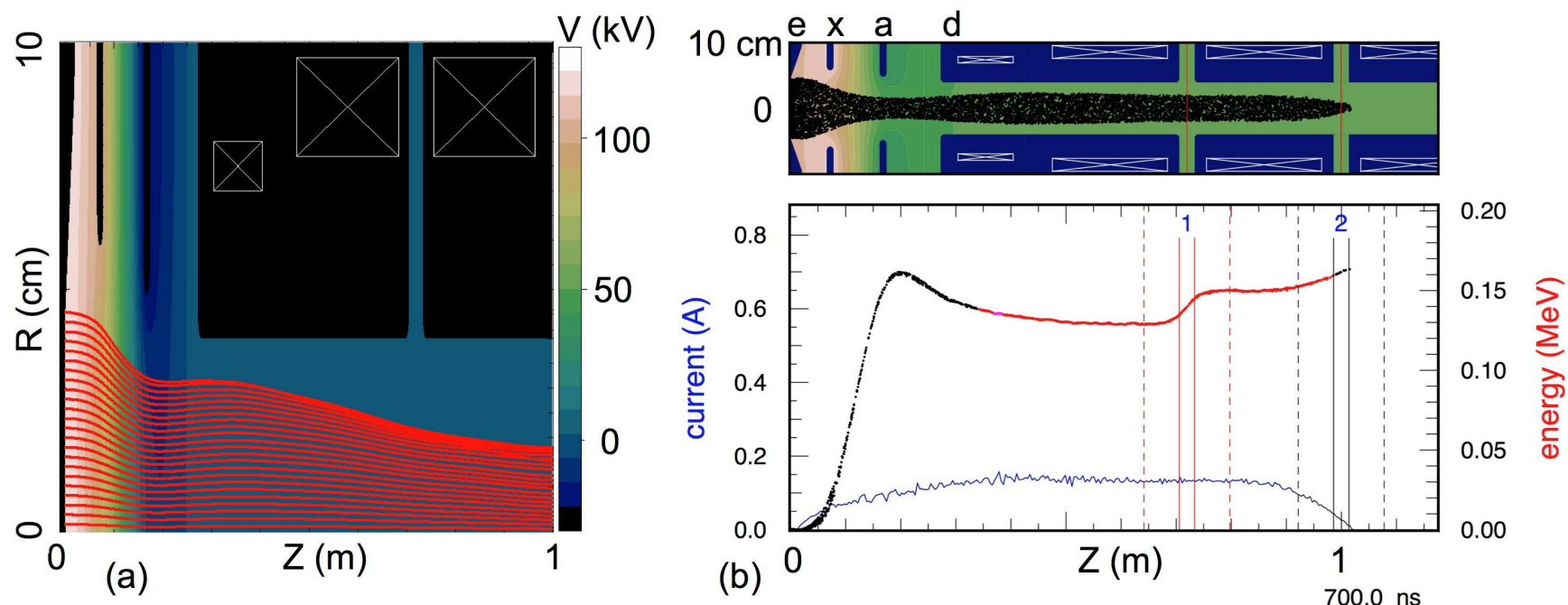

FIG. 6: (color online) Warp simulations of an injector configuration: (a) as designed using steady-flow "gun" model, showing trajectories (stretched ordinate); (b) snapshot of beam at $700 \mathrm{~ns}$ (true aspect ratio), and profiles of current and kinetic energy. Electrodes are: emitter ("e"), extractor ("x"), accel ("a"), and decel ("d"). The "accel-decel" pair provides transverse focusing.
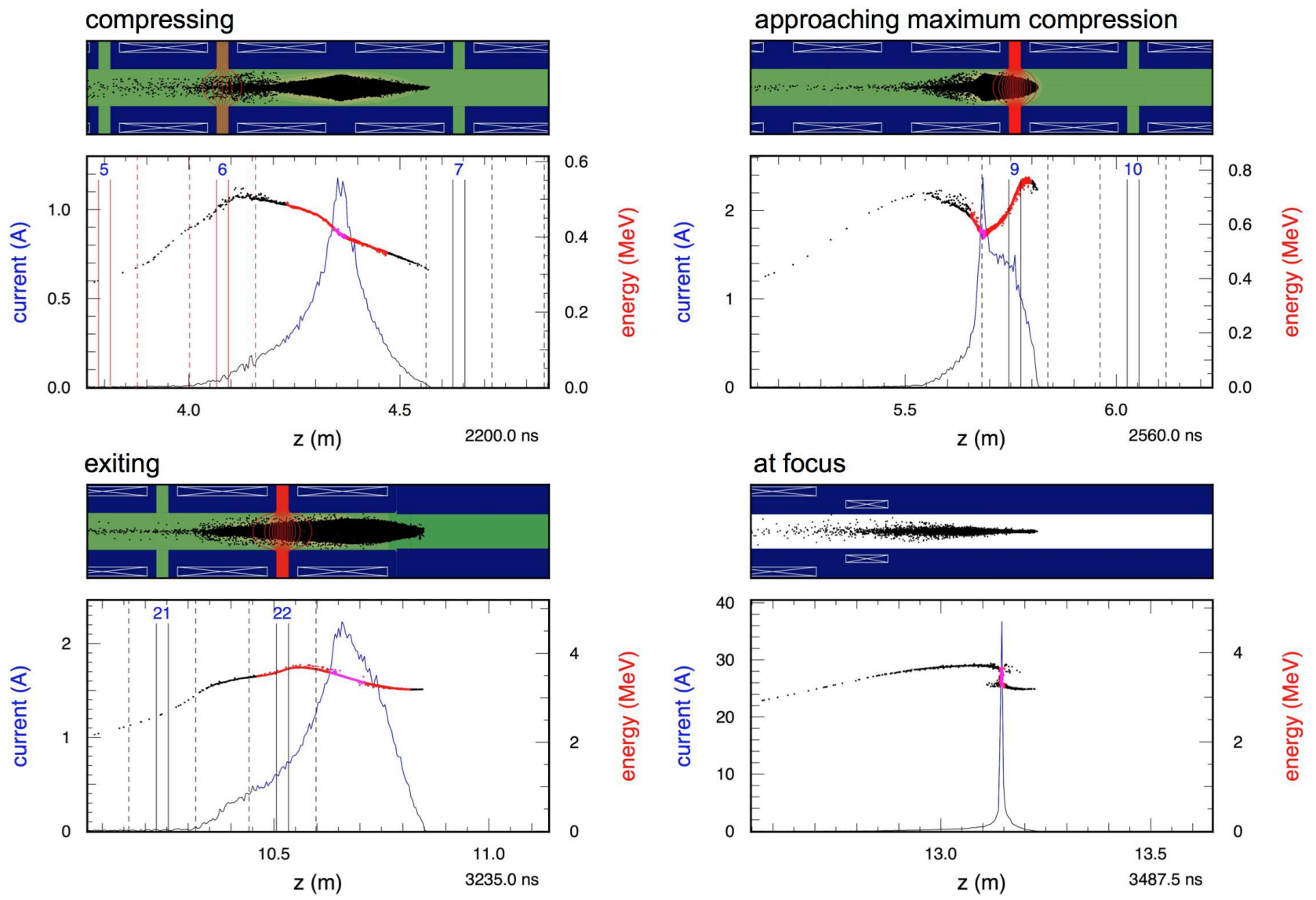

FIG. 7: (color online) Snapshots from a video sequence generated using Warp in $(r, z)$ geometry (enhanced online).

nonzero, but the field in the gap is slowly varying in time and is divergence free and nearly curl free, so that for computational purposes an electrostatic approximation is excellent.

Figure 7 shows a few snapshots from a Warp movie; the red lines in the active gaps are contours of the effective potential. The simulated energy deposition obtained from this run, with the 10-cm, 8-T solenoid from NDCXI about $24 \mathrm{~cm}$ upstream of the focal plane, is shown in Fig. 8. A stronger solenoid would produce a more com- 


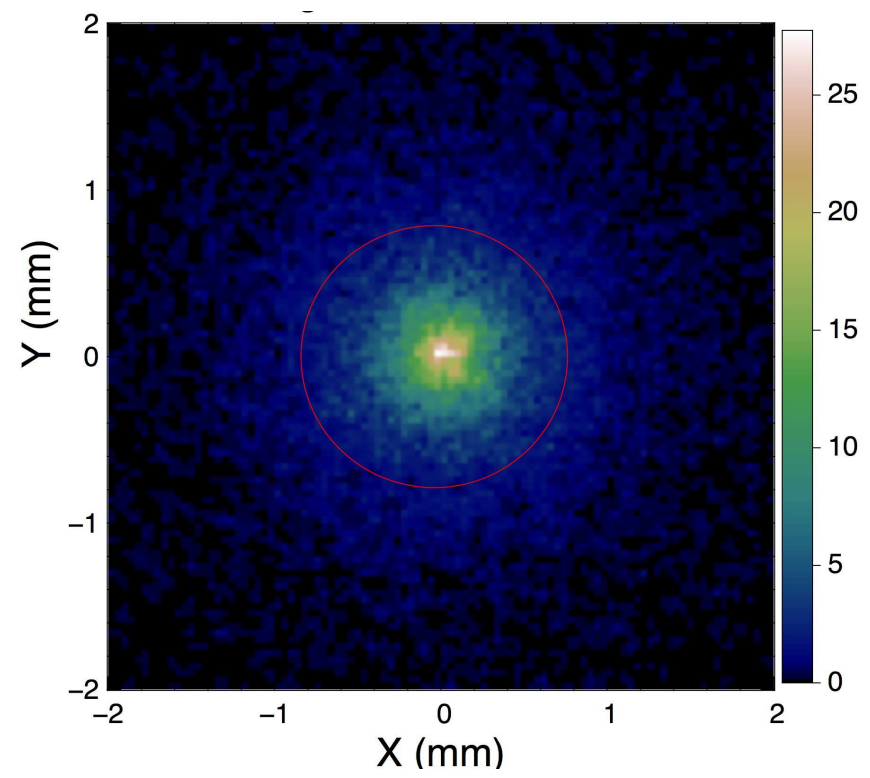

FIG. 8: (color online) Energy deposition into target plane at $z=13.15 \mathrm{~m}$ from Warp in $(r, z)$ geometry; colorbar denotes $\mathrm{J} / \mathrm{cm}^{2}$; semi-axes of ellipse equal RMS extents in $x$ and $y$.

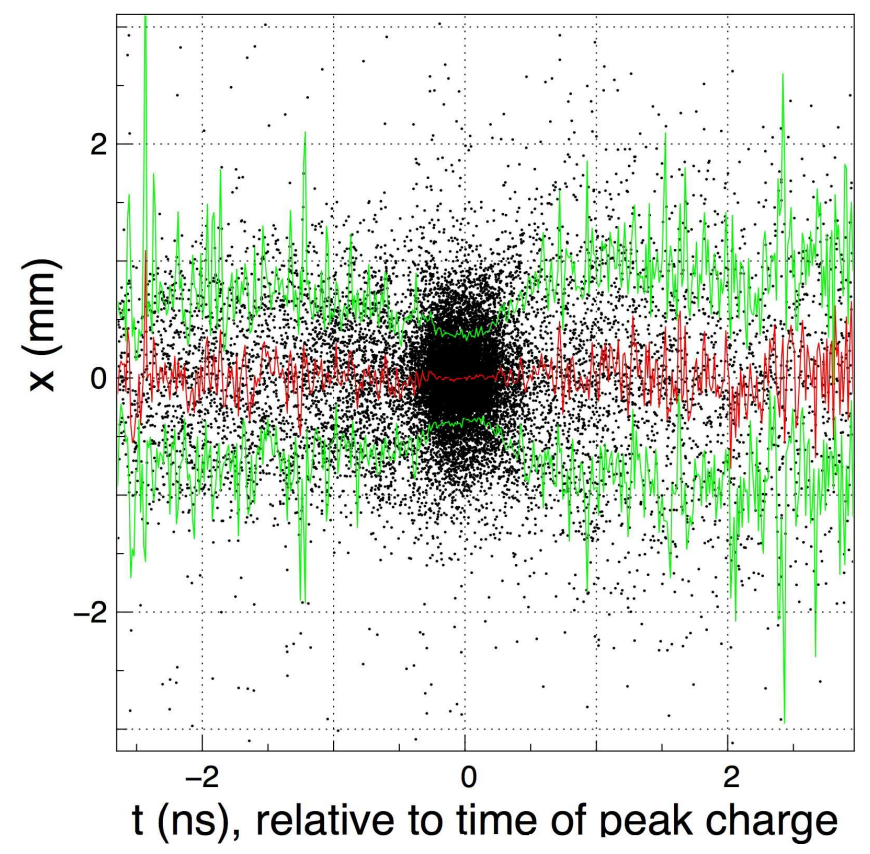

FIG. 9: (color online) $(x, t)$ phase space at target plane from Warp in $(r, z)$ geometry. Inner red line denotes centroid (fluctuations are due to noise); outer green lines denote RMS extent, relative to centroid.

pact spot. The combined longitudinal-transverse final focus is shown in Fig. 9, depicting the $(x, t)$ phase space at the target plane; the degree to which the beam is compact in space and time can be seen.

With random solenoid-end misalignments as large as the engineering-specification tolerance of $0.5 \mathrm{~mm}$ (corresponding to transverse offsets as well as pitch and yaw errors), both ASP and Warp simulations indicate good beam transmission through the accelerator. 3-D Warp simulations show that the degradation of the focal intensity is smooth and, for small errors, modest. Images from a 3-D simulation of a perfectly aligned system are shown in Fig. 10, and images from a similar simulation with 2-mm random displacements of the solenoid ends are shown in Fig. 11. The effects of misalignments on the focused beam intensity, as obtained from an ensemble of 3-D Warp runs with differing random errors, are shown in Fig. 12.

It is highly desirable to use steering dipole magnets to minimize both the corkscrew amplitude and the offaxis displacement. NDCX-II is mostly built using blocks of two or three cells separated by one or more nonaccelerating lattice periods; each of the latter includes a solenoid, steering dipoles for $x$ and $y$, and diagnostics to measure the beam centroid position. ASP results (Fig. 13) show that steering can be highly effective in NDCX-II, assuming good signal-to-noise ratios.

\section{DISCUSSION}

We have developed the physics design for a novel accelerator, using a new computational tool (ASP) which runs with sufficient speed to allow rapid NDCX-II design work, in tandem with an existing tool (Warp) which offers detailed multidimensional models. Computational aspects of this work, including ASP's 1-D space-charge field model with its simple correction for the finite radial geometry, self-adjusting waveforms that make maximal use of available volt-seconds and voltage, optimizations for waveform design and for beam steering, and an interactive very-high-level code framework based on Python (used in both ASP and Warp), have all worked out well. In view of the radial variation of the gap fields and ASP's approximate 1-D self-field, it was not clear at the outset that waveforms developed in ASP would be effective in accelerating, confining, and compressing the actual 3-D beam as modeled in Warp. We were pleased to see that our ASP-based design procedure works reliably, as may be inferred by inspection of Figs. 5 and 7 .

The beam manipulations employed in NDCX-II are themselves relevant to the physics of a driver accelerator for Heavy Ion Fusion power production. Driver beams will be space charge dominated both transversely and longitudinally, in the sense that quiescent propagation is achieved by balancing, primarily, the space charge forces and the applied focusing (confinement), with thermal pressure (emittance) a smaller contributor. The "bounce" employed in NDCX-II is, in essence, the technique for energy equalization envisioned for use near the target in a driver to enable focusing with minimal chromatic aberration. We believe NDCX-II will be the first experimental embodiment of this process. The machine will also enable a wide variety of studies of beam dynamics, both non-neutralized and neutralized.

This paper has described the physics design of the 

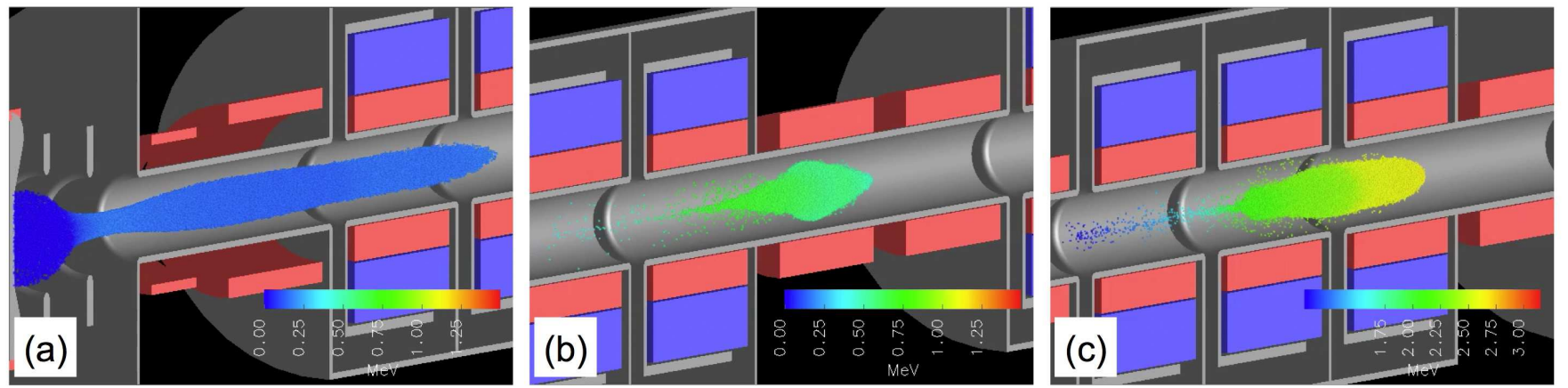

FIG. 10: (color online) Images from a video depicting the beam in a 3-D Warp simulation of NDCX-II; colorbars denote ion kinetic energy. The induction cores (blue color) are not shown to true scale - their radial extent is compressed by a factor of two, so as to allow the orientation of the induction cells to be seen. The red shapes denote the volumes occupied by the solenoids and their flux return channels. The system is assumed perfectly aligned, and the beam is shown (a) emerging from the injector; (b) after gap 8; and (c) passing through gap 16 (enhanced online).
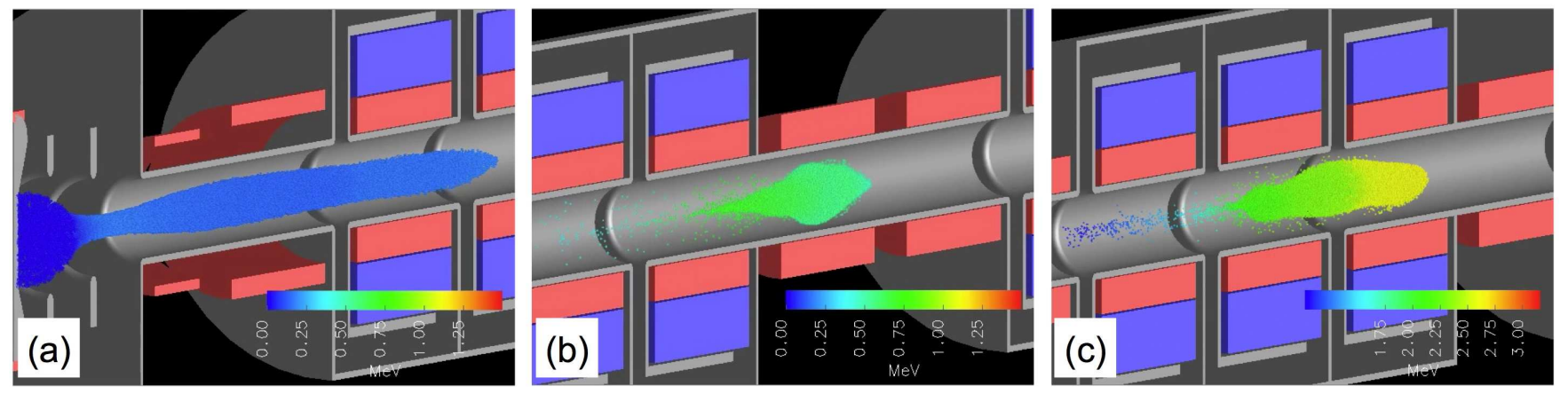

FIG. 11: (color online) Images from a video depicting the beam in a 3-D Warp simulation of NDCX-II; as in Fig. 10, but the system is assumed to be misaligned, with random transverse offsets of solenoid ends up to 2 mm (enhanced online).
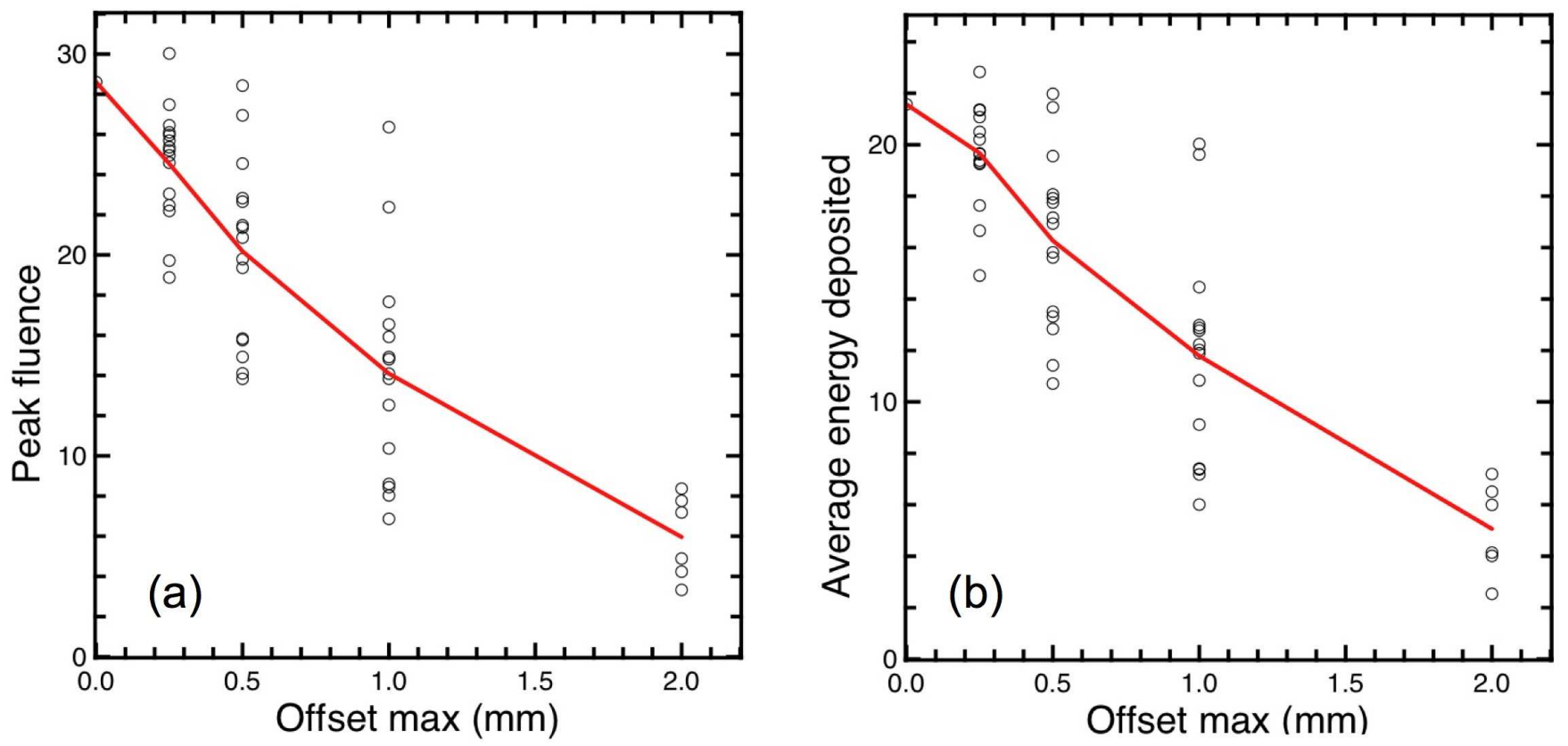

FIG. 12: Energy fluence $\left(\mathrm{J} / \mathrm{cm}^{2}\right)$ at focal plane versus amplitude of random offsets of solenoid ends (flat distribution with maximum shown on abscissa), from ensembles of 3-D Warp simulations (without steering dipoles): (a) peak deposition, time integrated; (b) average over circle of $0.1 \mathrm{~mm}$ diameter surrounding brightest spot, gated in time to include only central nanosecond around peak. 

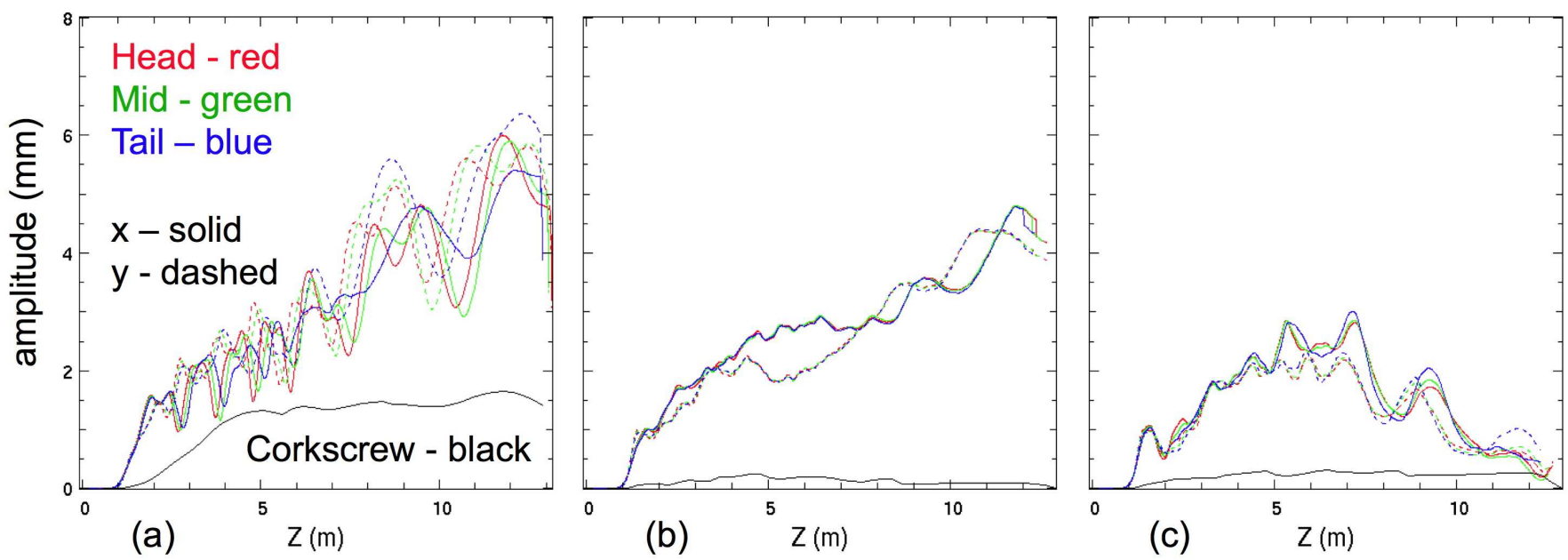

FIG. 13: (color online) ASP studies of steering (in an extended version of NDCX-II), showing RMS values of $x$ (solid lines) and $y$ (dashed lines) centroid coordinates vs. $z$ for head, middle, and tail particles, and the corkscrew amplitude; the results are averages over 20 simulations with differing random offsets of solenoid ends up to $1 \mathrm{~mm}$. Dipole magnets and sensors were placed in every fourth cell, and the penalty function was evaluated at the next sensor downstream from the dipole being varied. (a) without steering; (b) with steering optimization that penalizes corkscrew amplitude; (c) with steering optimization that penalizes corkscrew amplitude and beam offset, and constrains dipole fields to less than $100 \mathrm{G}$.

NDCX-II accelerator and has not attempted to cover many other important aspects of the full machine. Those aspects include the ion source and the pulsed power, as well as the neutralized drift line, final focusing solenoid, and target chamber (into all of which plasma must be injected, at sufficient density that the beam space charge is uniformly cancelled); see Ref. 20 for information on these and other related topics.

\section{Acknowledgments}

This work was performed under the auspices of the USDOE by LLNL under Contract DE-AC52-07NA27344, by LBNL under Contract DE-AC02-05CH11231, and by PPPL under Contract DE-AC02-76CH03073.
* Electronic address: af@llnl.gov; URL: http://hif.lbl.gov

1 B. G. Logan, F. M. Bieniosek, C. M. Celata, J. Coleman, W. Greenway, E. Henestroza, J. W. Kwan, E. P. Lee, M. A. Leitner, P. K. Roy, P. A. Seidl, J.-L. Vay, W. L. Waldron, S. S. Yu, J. J. Barnard, R. H. Cohen, A. Friedman, D. P. Grote, M. Kireeff Covo, A. W. Molvik, et al., Nucl. Instr. and Meth. A 577, 1 (2007).

2 S. M. Lidia, P. K. Roy, P. A. Seidl, W. L. Waldron, and E. P. Gilson, Proc. 2009 Particle Accelerator Conf. (PAC'09), Vancouver, May 2009, TU6PFP092; http: //www. JACoW. org.

3 D.P. Grote, A. Friedman, I. Haber, W. Fawley, J.-L. Vay, Nucl. Instr. and Meth. A 415, 428 (1998).

4 A. Friedman, J. J. Barnard, R. J. Briggs, R. C. Davidson, M. Dorf, D. P. Grote, E. Henestroza, E. P. Lee, M. A. Leitner, B. G. Logan, A. B. Sefkow, W. M. Sharp, W. L. Waldron, D. R. Welch, S. S. Yu, Nucl. Instr. and Meth. A 606, 6 (2009).

${ }^{5}$ W. M. Sharp, A. Friedman, D.P. Grote, E. Henestroza, M. A. Leitner, and W. L. Waldron, Nucl. Instr. and Meth. A 606, 97 (2009).

6 W. M. Sharp, A. Friedman, D.P. Grote, E. Henestroza, M. A. Leitner, and W. L. Waldron, Proc. 2009 Particle Accelerator Conf. (PAC'09), Vancouver, May 2009, TH5PFP072, http://www. JACoW. org.
7 A. Friedman, J. J. Barnard, R. H. Cohen, D. P. Grote, S. M. Lund, W. M. Sharp, A. Faltens, E. Henestroza, J-Y. Jung, J. W. Kwan, E. P. Lee, M. A. Leitner, B. G. Logan, J.-L. Vay, W. L. Waldron, R. C. Davidson, M. Dorf, E. P. Gilson, and I. Kaganovich, Proc. 2009 International Computational Accelerator Physics Meeting (ICAP'09), San Francisco, Sept. 2009, http://www. JACoW.org.

8 J. J. Barnard, G. J. Caporaso, S. S. Yu, and S. Eylon, Proc. 1993 Particle Accelerator Conf. (PAC'93), Washington, DC, May 1993, pp. 712-714, http://www. JACoW.org.

9 E. P. Lee (private communication, 2007); D. R. Welch, et al., Phys. Rev. ST Accel. Beams 11, 064701 (2008), Appendix. A.

10 Y-J. Chen, Nucl. Instr. and Meth. A 292, 455 (1990).

11 Y-J. Chen, Nucl. Instr. and Meth. A 398, 139 (1997).

12 See EPAPS supplementary material at [URL will be inserted by AIP] for: A. Friedman, "Some kinematic aspects of neutralized drift compression," LLNL Note LLNL-TR402447, March 2008.

13 A. B. Sefkow, R. C. Davidson, I. D. Kaganovich, E. P. Gilson, P. K. Roy, P. A. Seidl, S. S. Yu, D. R. Welch, D. V. Rose, and J. J. Barnard, Nucl. Instrum. and Methods A 577, 289297 (2007).

14 D. R. Welch, J. E. Coleman, P. A. Seidl, P. K. Roy, E. Henestroza, E. P. Lee, A. B. Sefkow, E. P. Gilson, T. C. 
Genoni, and D. V. Rose, PRST-AB 11, 064701 (2008).

15 I. D. Kaganovich, et al., Proc. 2009 Particle Accelerator Conf. (PAC'09), Vancouver, May 2009, http://www. JACow .org.

16 I. D. Kaganovich, "Physics of neutralization of intense charged particle beam pulses by a background plasma," submitted to Phys. Plasmas (2009); also Bull. Am. Phys. Soc. 54, 54 (2009).

17 R. C. Davidson, E. A. Startsev, M. Dorf, I. D. Kaganovich and H. Qin, Proc. 2009 Particle Accelerator Conf.
(PAC'09), Vancouver, May 2009, http://www. JACoW.org.

18 J.-L. Vay, P. Colella, J. W. Kwan, P. McCorquodale, D. B. Serani, A. Friedman, D. P. Grote, G. Westenskow, J.-C. Adam, A. Heron, and I. Haber, Phys. Plasmas 11, 2028 (2004).

19 P. K. Roy, LBNL, private communication (2009).

20 Proc. 17th Int. Sympos. on Heavy Ion Inertial Fusion (HIF2008), Tokyo, Aug. 4-9, 2008, published as Nucl. Instr. and Meth. A 606, (2009). 